\title{
RFID based Tracking System
}

\author{
Prof. Gaurav G. Narkhede ${ }^{1}$, Akshay Kishor Langade ${ }^{2}$, KinalGaurang Mehta ${ }^{3}$, Shubhankar Abhay Kulkarni ${ }^{4}$ \\ Assistant Professor, Electronics and Telecommunications Engineering, MIT COE, Pune, India ${ }^{1}$ \\ BE Student, Electronics and Telecommunications Engineering, MIT COE, Pune, India 2,3,4
}

\begin{abstract}
Radio Frequency Identification (RFID) is coming, and it's bringing a streamlined revolution. When dealing with the tracking device, Radio Frequency Identification (RFID) is the latest phase in the decades, that can be used as an efficient tracker. The development of Tracking System using Radio Frequency Identification (RFID) Technology is Quite new but something that promising. This solution uses RFID technique for monitoring entry and exit of employees with their official assets (E.g. laptops). The Tracking System actually based on external database system that will provide the recorded information about the reader. Since the reader detected by the database, then the tracking system will process the data and will show the result of subject tracking.
\end{abstract}

Keywords: Radio Frequency Identification (RFID), Asset Security, Asset Tracking using RFID, Asset Management using RFID, Security.

\section{INTRODUCTION}

Security of assets is the most important point for any the manual checks can be bypassed thus saving the manual organization to avoid unnecessary loss of data and leakage check and the queue load.

of strategies. Hence asset has a lot of importance to the companies.

It is a well-known fact that as organizations grow larger, there needs to be greater emphasis on using automated methods to track, trace, log and report on movement of the most important assets of the organization.

Effective asset tracking starts with getting the foundation in place: the tools and systems that enable asset tracking and software solutions that streamline data collection and analysis. These asset tracking tips will help you select the best asset tracking tools and software to meet your company's needs. RFID Fixed Asset Tracking has been a compelling use of RFID in large Enterprises and Fortune 500 corporations.

RFID tracking has seen tremendous growth in the corporate sector. One of the key concerns is related to its movement in \& out of office usually on a daily basis. This makes Instruments susceptible to theft which not only is loss of asset but more importantly loss of valuable and at times sensitive data. It is important for organization to ensure that right person carries the right laptop into \& out of the facility.

This solution uses RFID technique for monitoring entry and exit of employees with their official laptops(devices). The purpose of this solution is to reduce huge queues at company gates and limit the manual checks only for visitors and for the employees carrying unapproved laptops.

Master HR database of the company will have each employee's ID (RFID based I Card) and Laptop Tag (RFID based tag adhered to the laptop). If an employee enters or exits company gates with approved laptop, then

\section{LITERATURE SURVEY}

Location Detection is very important function in many areas such as robotics, aviation etc. A new type of position detection technology and new market interest in this technology was heavily researched nowadays. RFID technology is an automatic identification technology that uses radio frequency Signals to identify, track, sort and detect a variety of objects including people, vehicles, goods and assets. RFID technology can track the movements of objects through a network of radio frequency enabled scanning devices over a distance of few meters. With such benefits, many researchers used RFID technology in position tracking or detection of people or the assets.

This system is cost efficient as compared to the use of GPS (Global Positioning System) for Location Tracking. And more Featured compared to the standard RFID systems which were used for Security systems because of the use of the UHF (Ultra High Frequency) RFID tags. It also provides us with Tracking within a small region. whereas this paper attempts to employ a passive solution (which is cheaper and more cost-effective), to serve the purpose.

\section{III.OVERVIEW OF RFID TECHNOLOGY}

A typical RFID system is made up of three components: RFID tags, Readers and host computer system. An RFID tag is small radio device that is also referred to as Transponder or radio barcode. It is comprising of silicon microchip. This smart tag is adhered to an object and read remotely to verify its identity, position. 


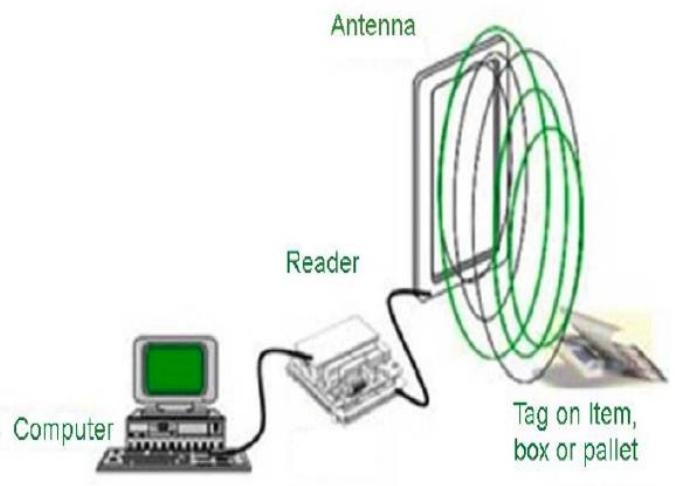

Fig. 1 Basic RFID System

A. Different RFID Types:

When you check your paper on a black-and-white hardcopy, please ensure that:

- Low Frequency (125/134 KHz): Most widely used for access control, asset tracking.

- High Frequency (13.56 MHz): Range is upto 1.5 meters. Used where

- Ultra-High Frequency (850 - 950 MHz): This frequency band offers the Longest read ranges of up to approximately 3 meters and high reading speed.

- Active RFID Tags:These tags are Battery Powered. They broadcast a signal to the reader and can transmit over a greatest distance $(100+$ meters $)$. The main application of these tags is to track the high value goods like vehicles and large containers of goods.

- Passive RFID Tags: They draw their power from the radio wave transmitted by reader. The reader transmits a low powered radio signal through its antenna to the tag, which in turn receives it through its own antenna to power the integrated circuit. Passive tags can transmit information over shorter distances (typically 3 meters or less) than Active tags. Also have a smaller memory capacity but ideal for tracking the assets.

\section{IV.SYSTEM OVERVIEW}

The basic aim of the system is to Track the official assets assigned to the authorised official of an Organisation so that important, valuable and confidential data should be safe.

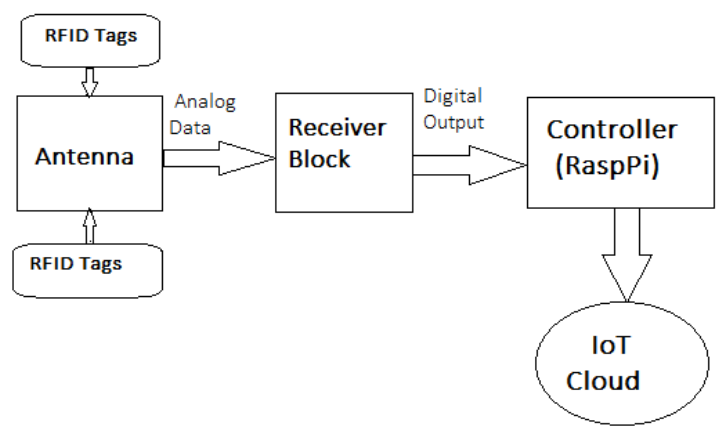

Fig. 2. Block diagram of RFID based tracking system
The block diagram shows that the Instruments/assets are tracked and we can have the complete record of the instruments/assets and the people which are registered with the organization. The instruments/assets can also be mapped to the person they have been assigned to, so that the particular device is always checked when moving out of the campus or the range of the receiver, to whether the person it is assigned to is also with it or not.

There are many possible cases for the instrument and the person it is assigned to:

- A registered person taking his own registered device

- A registered person taking other registered device.

- A registered person with an unregistered device/ without any device etc.

We are using an RFID in UHF range along with its tags so that there is no need to go to a particular place where their Reader is located.

\section{SYSTEM COMPONENETS}

A. RFID TAG

RFID tags has a significant contribution in the overall performance of the RFID based tracking system. The attributes such as Form factor, Price, read range etc. is considered while choosing the best tag for the application. Also we have to identify the very basic differences between various available UHF tags.

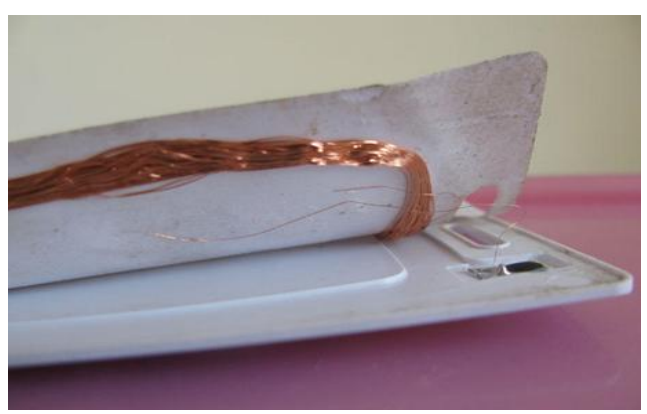

Fig. 3. UHF RFID Tag

\section{B. Antenna}

Choosing a proper UHF RFID antenna could be tedious and confusing while working with RFID first time. Hence we consider the Technical Specifications to clarify the picture. The following specifications are to be mainly considered while choosing an efficient RFID Antenna:

- $\quad$ Frequency Range

- Gain

- Polarization

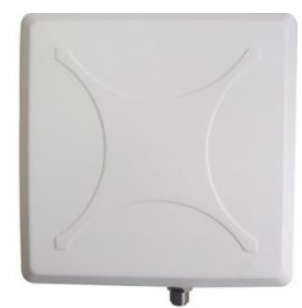

Fig 4 UHF RFID Antenna 
C. Receiver Block

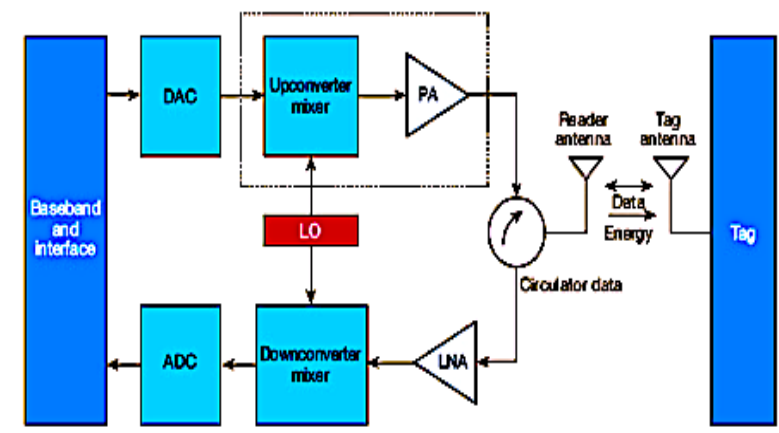

Fig. 5. RFID Reader Block Diagram

The principle of Electromagnetic Wave Propagation is basically used in a passive RFID system. The basic functioning of the RFID Reader is to transmit the EM waves which are later received by the antenna present in the Tags. The tag antenna is Impedance mismatched with respect to the Reader antenna to create reflections which are received and observed by the reader.

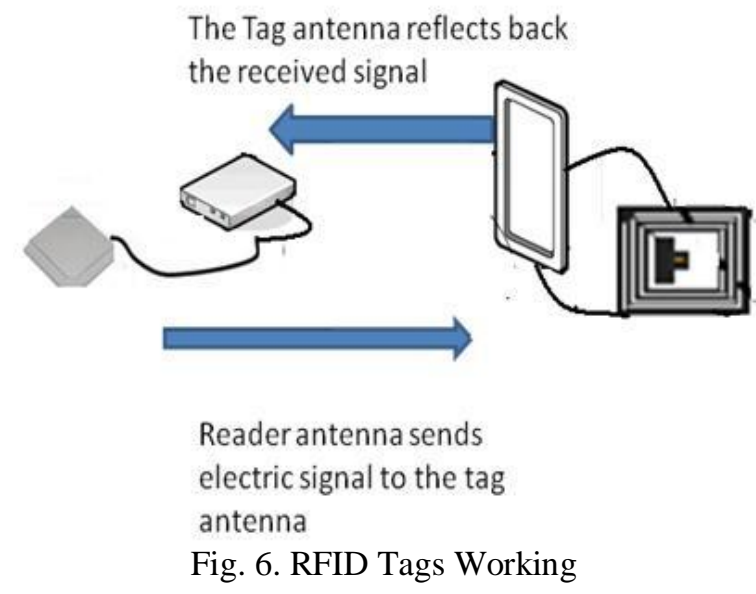

D. Raspberry Pi

The Raspberry Pi 3:

1. A $1.2 \mathrm{GHz}$ 64-bit quad-core $\mathrm{CPU}$

2. $802.11 \mathrm{n}$ Wireless LAN

3. 1GB RAM

4. 4 USB ports

5. Micro SD card slot.

\section{VI.SYSTEM IMPLEMENTATION}

Implementation of the system involves 2 main parts which can be broadly classified as:

\section{A. Hardware Implementation}

The following flowchart explains the working of the hardware implementation which uses the Python 3.5.2 platform to communicate with the UHF RFID reader as well as process the reader output and monitor the in or Out movement of the asset adhered with the respective assigned RFID tag and creates the file for the same.

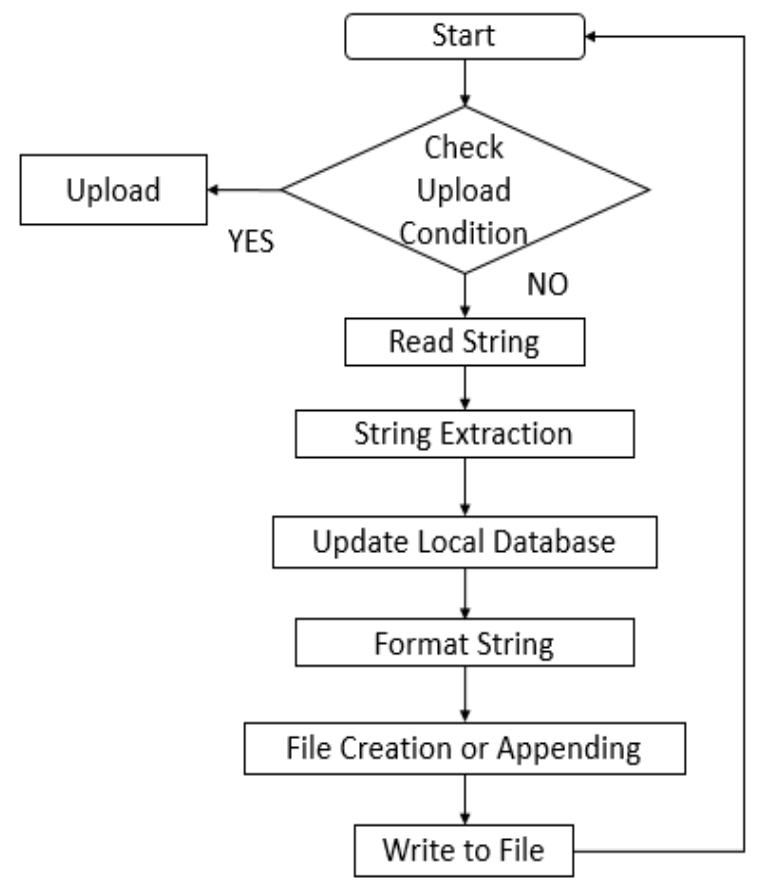

Fig. 7. Python code flowchart

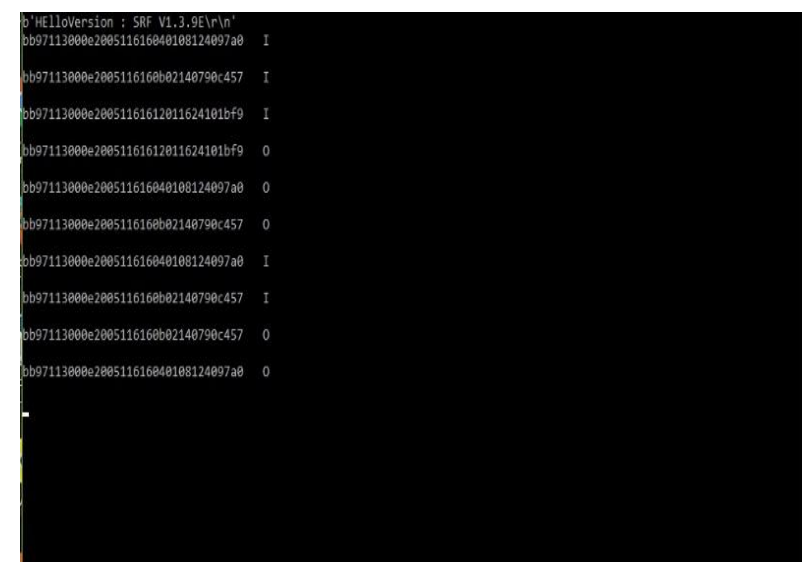

Fig. 8 Tag detection on python

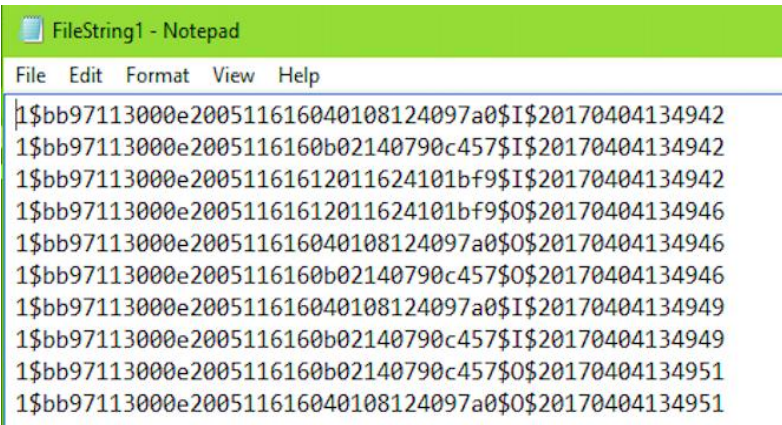

Fig. 9. File Contents

B. Software Implementation

This cron job will run on the server every-minute, which will detect uploaded files and enter the data into database. 


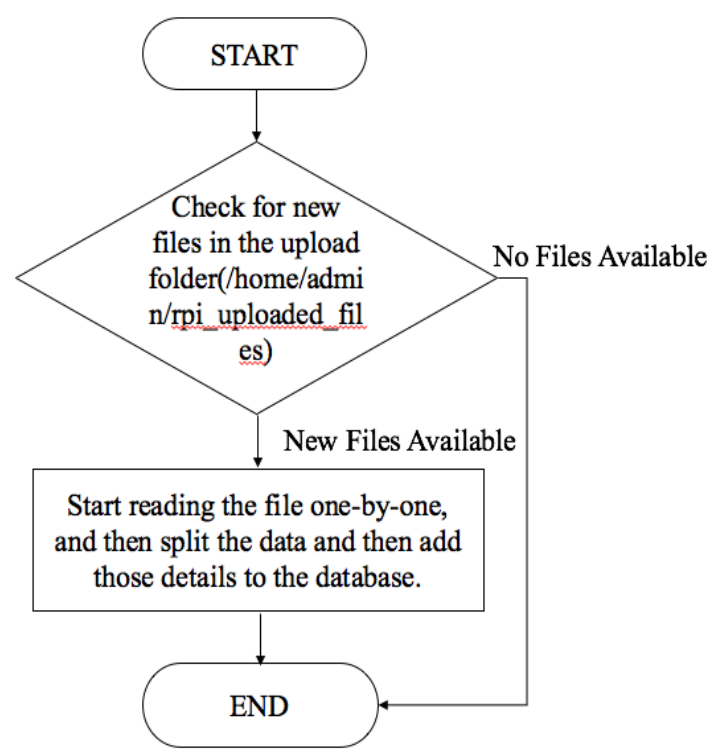

Fig. 10. Cron job flowchart

The following flow-chart explains the working after the data has been entered into the database. We query the database for the movements of the tags, and monitor the outward movement of the instrument/asset. The system alerts the administrator or the security personnel about the malicious activity. And then displays the updated movement table on the webpage

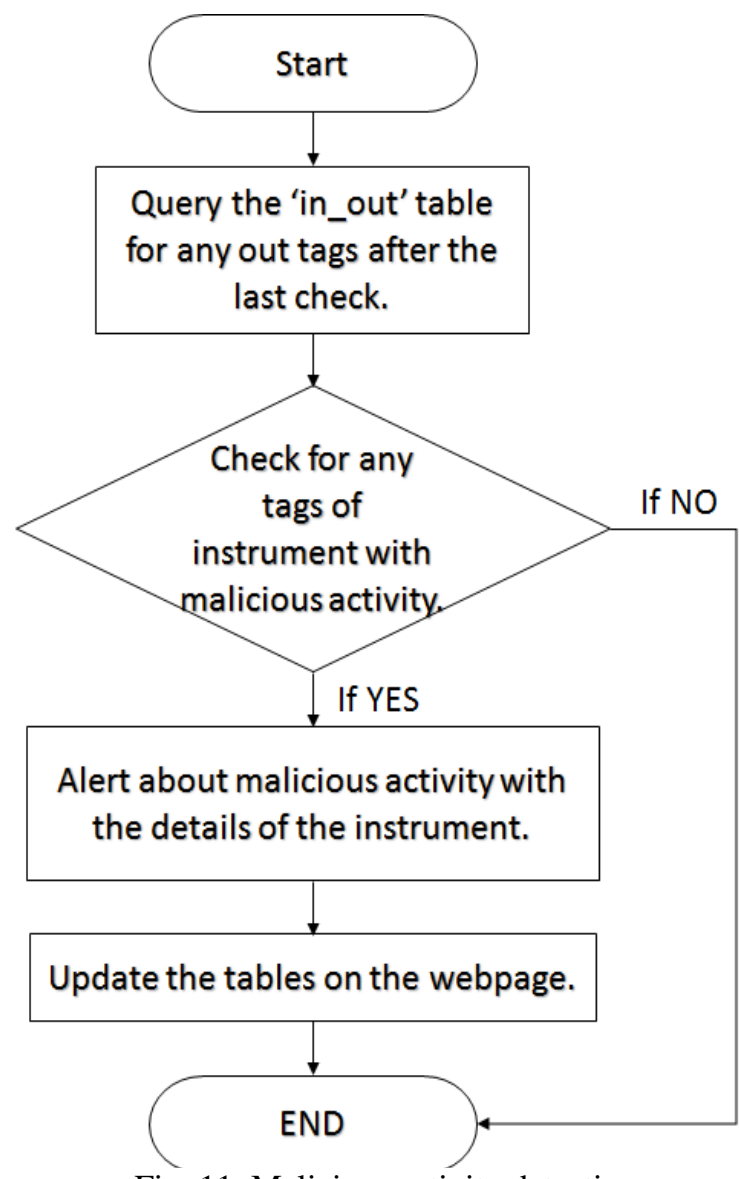

Fig. 11. Malicious activity detection
VII. RESULT AND DISCUSSION

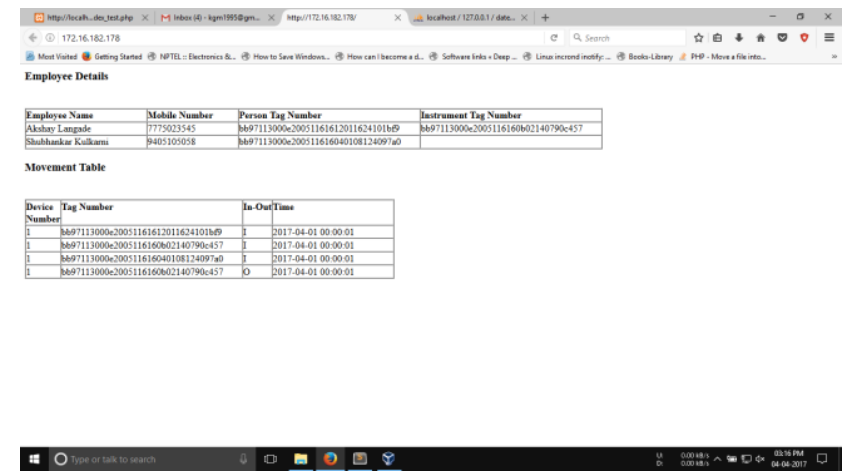

Fig. 12. Standard webpage output

The fig. 12 shows the output when there is no alert. This will show the employee details table and the movement table.

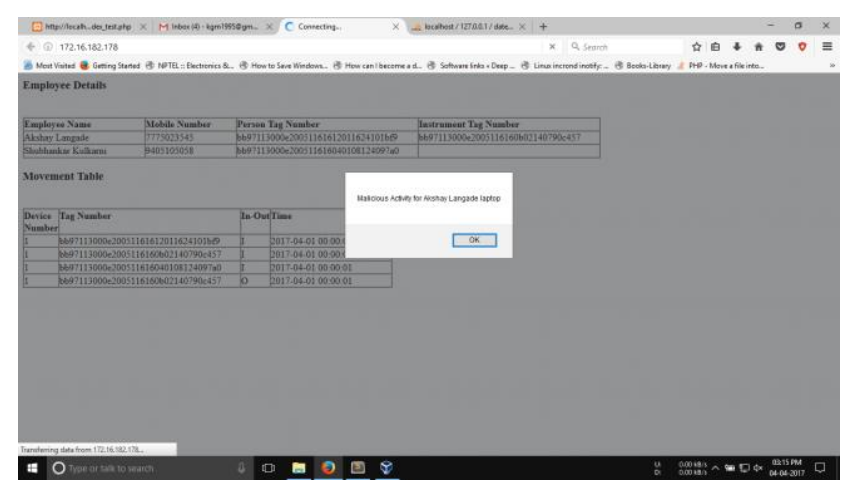

Fig. 13. Alert Output

The fig. 13 shows the alerting system when some malicious activity occurs.

\section{CONCLUSION}

The proposed system makes use of RFID technology to track the location of staff members and their respective laptops or instruments of any large organisation systematically. RFID systems uses emitted radio waves from the reader to transmit tag information wirelessly to a host computer. The tag information of the tags in the wireless range of reader gets stored in the server database. This indoor tracking system is based on the detection of a passive tag attached to an asset. Inhigh frequency passive tags, the distance up to which a tag can be detected is up to $10 \mathrm{~m}$. Higher frequency active tags give better range (up to few hundred meters). Passive tags used in this system are cost effective as compared to their active counterparts. Thus, the system provides a cost efficient solution for location tracking.

Strong Security of the Confidential data and valuable assets of the organization are achievable within simple Asset tracking system design which uses RFID technology.In this project report we described about RFID, 
principle, brief system design and implementation of RFID based tracking system, also benefits using of the system and RFID etc.

\section{IX.FUTURE SCOPE}

Some minor changes in the software can be made, and the system can also be used as attendance system in schools and colleges.

The design of the system is scalable at the cost of response time. System can be used on very large campuses with multiple companies in a campus.

\section{REFERENCES}

[1] P. Youngsu, W.L. Je, K. SangWoo, "Improving position estimation on RFID tag floor localization using RFID reader transmission power control," Robotics and Biomimetics, 2008. ROBIO 2008. IEEE International Conference (2009) 1716-1721

[2] F. Manzoor, H. Yi, K. Menzel, "Passive RFID-based indoor positioning system: an algorithmic approach," RFID-Technology and Applications (RFID-TA), IEEE International Conference (2010) 112-117

[3] H. Koyuncu, S.H. Yang, "A survey of indoor positioning and object locating systems," IJCSNS Int. J. Comput. Sci. Network Secur., 10 (5) (2010), pp. 121-128. Y. Yorozu, M. Hirano, K. Oka, and Y. Tagawa,

[4] M. Brandl, , T. Posnicek , K. Kellner Position, "Estimation of RFID- based sensors using SAW compressive receivers," (2016)

[5] S.L. Ting, S.K. Kwok, Albert H.C. Tsang and George T.S. Ho, "The Study on Using Passive RFID Tags for Indoor Positioning" International Journal of Engineering Business Management, 2011 